\title{
Evolución paleoambiental de una turbera finiholocena en el sector suroccidental del Parque Nacional de Doñana (S.O. España)
}

\section{Palaeoenviromental evolution of a Late Holocene peat bog in the southwestern sector of the Doñana National Park (SW Spain)}

\author{
M. Abad ${ }^{1}$, A.F. Muñoz ${ }^{2}$, M.L. González-Regalado ${ }^{3}$, F. Ruiz'3,4,7, J. Rodríguez-Vidal ${ }^{3,7}$, \\ L.M. Cáceres ${ }^{3,7}$, M.I. Carretero ${ }^{5}$, G. Monge ${ }^{5}$, M. Pozo ${ }^{6}$, M.I. Prudencio ${ }^{4}$, M.I. Dias ${ }^{4}$, \\ R. Marques ${ }^{4}$, T. Izquierdo ${ }^{1}$, J. Tosquella ${ }^{3}$, V. Romero ${ }^{3}$ \\ ${ }^{1}$ Universidad de Atacama. Avda. Copayapu 485, Copiapó, Chile. ORCID ID: http://orcid.org/0000-0003-1930-2650. \\ ${ }^{2}$ Departamento de Ciencias Integradas, Universidad de Huelva, Avda. Tres de Marzo, s/n, 21071-Huelva, España. \\ ORCID ID: http://orcid.org/0000-0002-1647-357X \\ ${ }^{3}$ Departamento de Ciencias de la Tierra., Universidad de Huelva, Avda. Tres de Marzo, s/n, 21071-Huelva, España. Email: \\ ruizmu@dgyp.uhu.es, ORCID ID: http://orcid.org/0000-0002-2626-9142, ORCID ID: http://orcid.org/0000-0002-7110-3133; \\ ORCID ID: http://orcid.org/0000-0002-9475-3307, ORCID ID: https://orcid.org/0000-0002-1381-2476, ORCID ID: http://orcid. \\ org/0000-0001-7951-0561, ORCID ID: http://orcid.org/0000-0002-6879-0243 \\ ${ }^{4}$ C2TN, Instituto Superior Técnico, Universidad de Lisboa, 2695-066-Bobadela, Portugal. ORCID ID: https://orcid.org/0000- \\ 0003-2311-1806, ORCID ID: http://orcid.org/0000-0002-7033-0502, https://orcid.org/0000-0001-6239-5456 \\ ${ }^{5}$ Departamento de Cristalografía, Mineralogía y Química Agrícola, Universidad de Sevilla, C/ Profesor García González, \\ 1, 41012-Sevilla, España. ORCID ID: http://orcid.org/0000-0002-7277-0851. ORCID ID: http://orcid.org/0000-0002- \\ 3171-3439 \\ ${ }^{6}$ Departamento de Geología y Geoquímica, Universidad Autónoma de Madrid, 28049-Madrid, España. ORCID ID: http://orcid. \\ org/0000-0002-6706-440X \\ ${ }^{7}$ Centro de Investigación en Patrimonio Histórico, Cultural y Natural (CIPHCN), Universidad de Huelva, Facultad de \\ Humanidades, Avda. Tres de Marzo s/n, 21071-Huelva, España.
}

\section{RESUMEN}

El análisis multidisciplinar de un perfil descubierto por las tormentas invernales en el litoral del Parque Nacional de Doñana ha permitido reconstruir la evolución de su sector occidental durante el Holoceno superior. Se han definido 3 fases que reflejan el paso desde una marisma de aguas salobres (FA-1) a una laguna de aguas dulces transformada en turbera (FA-2), para finalizar con el depósito de cordones dunares (FA-3).

Palabras clave: Facies sedimentarias; evolución paleogeográfica; Holoceno superior; Doñana; S.O. España.

\section{ABSTRACT}

The multidisciplinary analysis of a profile discovered by the winter storms on the coast of the Doñana National Park has allowed us to reconstruct the evolution of its western sector during the Late Holocene. Three phases have been defined, which reflect the transition from a brackish water marsh (FA-1) to a freshwater lagoon transformed into peat bog (FA-2), to end with the deposit of dune systems (FA-3).

Keywords: Sedimentary facies; palaeogeographical evolution; Late Holocene; Doñana; S.W. Spain.

Recibido el 13 de noviembre de 2018; Aceptado el 12 de febrero de 2019; Publicado online el 29 de marzo de 2019

Citation / Cómo citar este artículo: M. Abad et al. (2019). Evolución paleoambiental de una turbera finiholocena en el sector suroccidental del Parque Nacional de Doñana (S.O. España). Estudios Geológicos 75(1): e087. https://doi.org/10.3989/egeol.43417.514

Copyright: ( $\odot 2019$ CSIC. This is an open-access article distributed under the terms of the Creative Commons Attribution-Non 4.0 International License. 


\section{Introducción}

En los últimos años, han proliferado los estudios sobre el registro holoceno de medios costeros, con especial atención a la incidencia de los cambios climáticos y las fluctuaciones del nivel del mar sobre ellos (Cohen et al., 2016; Fanget et al., 2016; Araujo et al., 2017; Chase \& Quick, 2018). Sus resultados suelen basarse en el análisis pluridisciplinar de testigos continuos o secciones superficiales, que a menudo incluye la identificación de diatomeas o polen para poder deducir su evolución paleogeográfica y ambiental (Anderson, 2000; Leventer et al., 2010; Prieto et al., 2017; Blázquez et al., 2017; Quick et al., 2018).

Otras investigaciones se han centrado en los eventos de alta energía (tormentas, tsunamis). Algunas de ellas inciden en sus periodicidades y duración (Castele et al., 2018; Pouzet et al., 2018) en tanto que otras se centran en las evidencias geológicas asociadas (Ruiz et al., 2013; Rubin et al., 2017; Putra, 2018) o en la producción de mapas de riesgos de las posibles zonas inundables (AnielQuiroga et al., 2017; Arias et al., 2017; AguirreAyerbe et al., 2018).

En el suroeste de la Península Ibérica se han intensificado estos estudios en las últimas décadas. Sus temas principales han sido la identificación de las fases de progradación y erosión costeras (p.e. Zazo et al., 1994; Dabrio et al., 1999, 2000), la evolución paleoambiental y paleogeográfica de sus estuarios y parajes naturales (p.e. Borrego et al., 1999; Ruiz et al., 2004, 2005a; Borja, 2013) o el registro geológico de tsunamis y tormentas (p.e. Alonso et al., 2004; Lario et al., 2010; Rodríguez Vidal et al., 2011a,b; Ruiz et al., 2008, 2013). Algunos de ellos se han centrado en el análisis evolutivo holoceno de distintas turberas que jalonan su litoral (p.e. Menéndez Amor \& Florschütz, 1963; Stevenson, 1985b).

En este trabajo, se efectúa un análisis granulométrico, estratigráfico, mineralógico y paleontológico de un perfil costero situado en el sector occidental del Parque Nacional de Doñana. Este estudio multidisciplinar permite efectuar una reconstrucción paleoambiental de esta zona durante el Holoceno superior.

\section{El Parque Nacional de Doñana}

\section{Caracterización geomorfológica básica}

El Parque Nacional de Doñana (S.O. de España) se encuentra en la orilla occidental del estuario del río Guadalquivir, con una extensión de 54.251 ha. Constituye uno de los mayores humedales de Europa y se compone principalmente de marismas fluvio-mareales con una modesta topografía $(<2-3 \mathrm{~m})$. Estas marismas están drenadas por dos cauces principales (río Guadiamar y arroyo Madre de las Marismas) y numerosos canales finalizadores, con sus márgenes actuales ocupados por levees arcillosos y cheniers.

Las zonas internas del Parque están protegidas por dos flechas arenosas (Doñana-La Algaida) que incluyen sistemas activos de dunas dispuestos en alineaciones estrechas $(<100 \mathrm{~m})$, alargadas $(1-2 \mathrm{~km})$ y con alturas máximas que rondan los $30 \mathrm{~m}$. En la zona interna de la flecha de Doñana se observan crestas arenosas y cheniers con una dirección NE-SW dispuestos sobre las marismas y enraizadas en la propia flecha. En esta zona también se disponen un conjunto de lagunas costeras, con unas propiedades hidrológicas, geoquímicas, mineralógicas y edafológicas especiales (Recio et al., 2009). Muchas de ellas son semipermanentes, de escasa profundidad $(<3 \mathrm{~m})$, presentan bajas conductividades $(<10 \mathrm{mS} / \mathrm{cm}$ excepto en épocas de sequía) un $\mathrm{pH}$ levemente ácido a muy alcalino (5-11) y se localizan en suelos eólicos muy permeables (Vanney \& Menanteau, 1985; Toja et al., 1991).

\section{Registro geológico holoceno}

El registro del sector meridional del Parque Nacional de Doñana ha sido analizado recientemente con mayor profundidad en base a numerosos sondeos realizados por el Instituto Geológico y Minero de España (Salvany \& Custodio, 1995; Salvany et al., 2010). De forma sintética, estos sondeos han puesto de manifiesto la presencia de un sustrato neógeno compuesto por la Formación Arenas de Huelva (Civis et al., 1987) 
y la Formación Arenas de Bonares (Mayoral \& Pendón, 1986-7), sobre las que se disponen un manto aluvial (Formación Arenas y Gravas de Almonte; Salvany et al., 2010), unidades aluviales, arenas eólicas (Formación Arenas de El Abalario; Salvany et al., 2010) y, localmente, flechas litorales.

En el sur peninsular, las barreras arenosas han evolucionado mediante una alternancia de fases de progradación con otras de erosión o no depósito, condicionadas por variaciones climáticas (Zazo et al., 2008). En este sentido, Zazo et al. (1994) han reconocido cuatro etapas de progradación $\left(\mathrm{H}_{1}\right.$ : 6500-4400 cal. yr $\mathrm{BP} ; \mathrm{H}_{2}$ : 4200-2550 cal. yr BP; $\mathrm{H}_{3}: 2300-800$ cal. yr. BP; $\mathrm{H}_{4}$ : 500 cal. yr BP hasta la actualidad), de las cuáles las dos últimas están representadas en la flecha de Doñana.

\section{Hidrodinámica}

Los principales procesos hidrodinámicos están controlados por el régimen fluvial, los aportes mareales, el oleaje dominante procedente del suroeste y las corrientes de deriva litoral, de sentido W-E. El río Guadalquivir tiene un régimen muy irregular, con una media anual de $185 \mathrm{~m}^{3} / \mathrm{s}$ y un máximo algo superior a los 1.000 $\mathrm{m}^{3} / \mathrm{s}$ (Vanney, 1970; Menanteau, 1979). El régimen mareal es mesomareal y semidiurno, con un rango medio mareal de 3,6 m (Borrego et al., 1993).

\section{Eventos de alta energía: pasado y presente}

La costa suratlántica de la Península Ibérica es un área de baja probabilidad tsunamigénica (Campos, 1991; Reicherter, 2001), con 16 tsunamis registrados entre el 218 a.C. y 1900 (Galbis, 1932). El registro geológico en las zonas costeras y cuencas oceánicas próximas ha sido objeto de numerosos trabajos en las últimas dos décadas, que han permitido conocer las formaciones sedimentarias derivadas de su acción erosiva-deposicional (p.e. Luque et al., 2001, 2002; Scheffers \& Kelletat, 2005; Gutiérrez-Más et al., 2009;
Rodríguez Vidal et al., 2011a,b; Gutiérrez-Más \& Más, 2013; Ruiz et al., 2013; Font et al., 2013; Alonso et al., 2015) y ampliar este registro hasta hace unos 7.000 años (Gràcia et al., 2010; Lario et al., 2011).

Este litoral está sujeto también a la acción de fuertes temporales invernales. Periodos prolongados de tormentas coinciden con fases negativas de la NAO (North Atlantic Oscillation) y la AO (Artic Oscillation) (Anfuso et al., 2016). En el litoral de Doñana, el estudio de series temporales de tormentas invernales ha permitido definir dos ciclos de periodicidad de estos eventos energéticos (2-3 y 6-7 años), con nueve grandes periodos de tormentas entre 1960 y 1999 (Ruiz et al., 2005b).

\section{Metodología}

\section{Trabajo de campo}

Las tormentas invernales de 2010 ocasionaron la erosión de la playa del sector suroccidental del Parque Nacional de Doñana (Fig. 1, A), próximo a la localidad de Matalascañas (Huelva, S.O. de España). Esta erosión provocó la aparición de un sustrato arcilloso-turboso a lo largo de más de un centenar de metros, situado en el límite de la pleamar.

En una primera fase, se determinaron las distintas facies presentes en un perfil perpendicular a la costa (Fig. 1, B-D). A continuación se extrajo1 kg de muestra en cada uno de los cuatro niveles (Fig. 1, C: DN-1 a DN-4) representativos de las dos facies más extendidas en el perfil para su análisis textural, mineralógico, micropaleontológico y cronoestratigráfico.

\section{Análisis granulométrico}

El análisis granulométrico de la fracción mayor de $100 \mu \mathrm{m}$ se realizó mediante tamizado en húmedo. Previamente las muestras se dispersaron en agua mediante agitación durante dos horas, empleando hexametafosfato sódico como agente dispersante. La distribución granulométrica de la fracción menor de $100 \mu \mathrm{m}$ se 


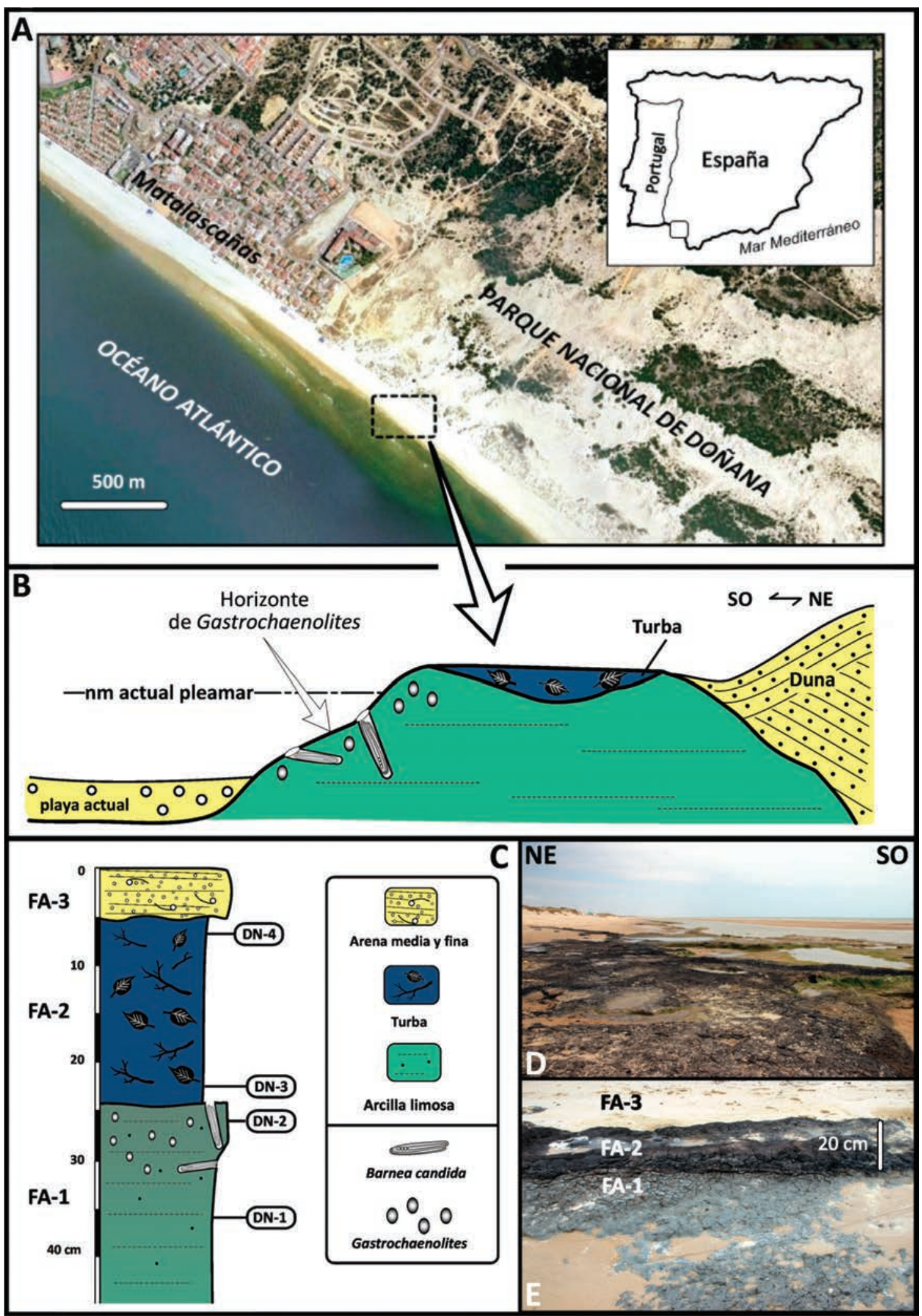

Figura 1.-A. Situación geográfica del perfil estudiado. B. Corte geológico del perfil. C. Columna litoestratigráfica con las facies diferenciadas. D. Vista general del perfil. E. Corte sintético con las tres facies distinguidas. 
determinó mediante fotosedimentación, utilizando un equipo (MicromeriticsR SediGraph 5100 ET).

\section{Análisis mineralógico}

Se analizaron cuatro muestras de los distintos niveles diferenciados mediante difracción de rayos $\mathrm{X}$ para obtener la mineralogía total. El equipamiento usado fue un difractómetro Philips PW 1130/90, con radiación $\mathrm{K} \alpha$ de $\mathrm{Cu}$ y un filtro de Ni a 20 mA y $40 \mathrm{kv}$. La fracción menor de $2 \mu \mathrm{m}$ fue separada por un método estándar de sedimentación (Barahona, 1974).

\section{Análisis macropaleontológico}

\section{Bivalvos}

Se realizó un estudio morfométrico de Barnea candida (L.), el único bivalvo hallado en el perfil, para deducir la edad de los ejemplares presentes. Además, se estudió la distribución y densidad de sus perforaciones (Gastrochaenolites isp.), de acuerdo con la metodología de Pinn et al. (2005).

\section{Estructuras macroscópicas vegetales}

Se analizaron 3 fragmentos de $5 \mathrm{~g}$ de turba (DN-4) recogidos en distintas zonas del afloramiento. En la lupa binocular se disgregó cada porción, con la observación y clasificación de las estructuras macroscópicas presentes.

\section{Análisis micropaleontológico}

Polen

A continuación se efectuó el análisis micropaleontológico de los cuatro niveles muestreados, con la determinación de la abundancia y diversidad de polen mediante una cámara de Neubauer. Para cada nivel (DN-1 a DN-4) se extrajeron 4 muestras (16 en total), que fueron disueltas en agua y homogeneizadas para proceder al contaje de la cantidad de granos de polen y de diatomeas (enteras o fracciones conspicuas) contenidas en 4 cámaras de $1 \mathrm{~mm}^{2}$ de superficie $\left(0,1 \mathrm{~mm}^{3}\right)$. Se procedió al contaje de polen para obtener su concentración media total en cada nivel.

Para cuantificar el contenido polínico, se extrajeron tantas muestras de $10 \mathrm{~mm}^{3}$ como fueron necesarias para obtener, al menos, 250 granos. Estas muestras se analizaron mediante un microscopio óptico a 1000 aumentos. Los granos de polen fueron identificados a partir de los datos de Valdés et al. (1987).

\section{Diatomeas}

El procedimiento fue similar para las diatomeas. La mayoría de las diatomeas de gran tamaño (p.e. Nitzschia, Ulnaria) presentaron un alto grado de fragmentación en todas las muestras, así como los individuos de todas las especies en los niveles DN-1 y DN-2. Este grupo fue identificado a nivel genérico a partir de Kelly et al. (2005), y utilizando diversas monografías para la determinación específica, unificando la nomenclatura de acuerdo con Guiry \& Guiry (2010).

\section{Ostrácodos y foraminíferos}

Se extrajeron $10 \mathrm{~g}$ de cada nivel muestreado, que fueron disueltos en agua con adición de Calgón y peróxido de hidrógeno. Esta solución fue tamizada a continuación $(125 \mu \mathrm{m})$. El residuo obtenido fue secado en estufa a una temperatura constante de $60^{\circ} \mathrm{C}$ y analizado mediante lupa binocular de hasta 80 aumentos. No se han observado ejemplares de ambos grupos en ninguna de las muestras analizadas.

\section{Dataciones}

Se realizaron dos dataciones en el Centro Nacional de Aceleradores de Sevilla. La primera muestra procedió del tramo superior de turba y fue calibrada por el programa IntCa109. El resultado final correspondió a edades calibradas (cal.) con un rango de 2 sigmas. La segunda muestra fue un ejemplar completo de Barnea candida extraído de una perforación (Gastrochaenolites isp.) realizada en la parte superior del nivel turboso, que no pudo ser calibrada debido a ser excesivamente reciente y quedar fuera del rango de calibración (ver apartado siguiente). 
Tabla 1.-Análisis granulométrico y mineralógico de los niveles muestreados (en \%).

\begin{tabular}{|c|c|c|c|c|}
\hline MUESTRAS & $\mathrm{DN}-1$ & $\mathrm{DN}-2$ & DN-3 & DN-4 \\
\hline \multicolumn{5}{|c|}{ GRANULOMETRÍA } \\
\hline Arena & 8.09 & 6.55 & 12.92 & 13.19 \\
\hline Limo & 80.29 & 75.23 & 71.66 & 74.35 \\
\hline Arcilla & 11.62 & 18.22 & 15.41 & 12.46 \\
\hline \multicolumn{5}{|c|}{ MINERALOGÍA TOTAL } \\
\hline Filosilicatos & 58 & 55 & 60 & 55 \\
\hline Cuarzo & 25 & 23 & 22 & 36 \\
\hline Plagioclasa & 7 & 9 & 4 & 2 \\
\hline Halita & 2 & 3 & 6 & 6 \\
\hline Pirita & 6 & 8 & 8 & \\
\hline Yeso & 2 & 2 & Identificado & 1 \\
\hline Otros & & $\begin{array}{l}\text { Silvina, } \\
\text { jarosita }\end{array}$ & $\begin{array}{l}\text { Silvina, } \\
\text { jarosita }\end{array}$ & \\
\hline \multicolumn{5}{|c|}{ FILOSILICATOS } \\
\hline Esmectita & 44 & 47 & 35 & \multirow{3}{*}{$\begin{array}{l}\text { Illita e } \\
\text { interestratificados } \\
\text { irregulares }\end{array}$} \\
\hline Illita & 48 & 40 & 52 & \\
\hline Caolinita & 8 & 13 & 13 & \\
\hline
\end{tabular}

\section{Resultados}

\section{Facies sedimentarias}

Los análisis de campo, granulométrico y mineralógico han permitido diferenciar tres facies en el perfil estudiado (Fig. 1, C).

Facies 1 (FA-1: limo arcilloso). Los $15 \mathrm{~cm}$ inferiores de este perfil están constituidos por limos arcillosos grisáceos masivos (Fig. 1, E), con porcentajes crecientes de arcilla y ligeramente descendentes de arena hacia techo (Tab. 1). Los filosilicatos son los minerales dominantes (Tab. 1: $55-58 \%$ ), con proporciones significativas de cuarzo (23-25\%) y, en menor medida, plagioclasa $(7-9 \%)$ y pirita $(6-8 \%)$. Dentro de los filosilicatos, esmectita $(44-47 \%)$ e illita (40-48\%) presentan similares porcentajes, en tanto que la caolinita aumenta hacia techo (hasta un 13\%).

A techo, esta facies presenta un gran número de perforaciones circulares en sección ecuatorial y cónicas en sección transversal (Fig. 1, E; Fig. 4, $\mathrm{N}$ : Gastrochaenolites isp.), ocupadas por numerosos ejemplares del bivalvo Barnea candida en posición natural. La longitud de estos ejemplares varía entre 15 y más de $40 \mathrm{~mm}$, con una mayoría de perforaciones ocupadas por individuos con menos de $25 \mathrm{~mm}$ de longitud.

Facies 2 (FA-2: turba). Esta facies está caracterizada por la presencia de numerosos restos vegetales dentro de una matriz limosa-arcillosa negra (Tab. 1), con un aumento importante de los porcentajes de arena (hasta un 13,2\%) en relación a FA-1. La mineralogía total está dominada por los filosilicatos $(55-60 \%)$, si bien el contenido en cuarzo aumenta hacia techo $(22-36 \%)$. La pirita solo se ha identificado en la base (Tab. 1: 8\%), en tanto que los contenidos en halita son relativamente constantes $(\sim 6 \%)$ en esta facies. Dentro de los filosilicatos, la principal diferencia mineralógica con FA-1 es el predominio de illita (52\%) sobre esmectita $(35 \%)$ en su base, así como la presencia de illita e interestratificados irregulares hacia techo.

Se han identificado cuatro tipos de estructuras macroscópicas vegetales en FA-2. La mayoría (12) corresponden a utrículos y cápsulas de ciperáceas del género Carex, que por su tamaño posiblemente pertenezcan a $C$. pseudocyperus L. También han aparecido tres aquenios de Eleocharis palustris (L.) Roem. \& Schult., dos esporangios de helechos con anillo de dehiscencia y dos semillas que por su morfología y anatomía podrían corresponder a alguna especie de la familia Poeaceae (subfamilia Panicoideae).

Facies 3 (FA-3: arena media y fina). Presenta una base erosiva dispuesta sobre FA-2. Está constituida por arenas finas a medias amarillentas, bien clasificadas, ricas en cuarzo y feldespatos y con escaso contenido limo-arcilloso. Presenta una continuidad lateral con los cordones dunares situados hacia el norte del perfil estudiado (Fig. 1, D).

\section{Polen}

Se han determinado un total de 33 tipos polínicos en los cuatro niveles analizados, con un porcentaje de granos no identificables comprendido entre el 4,69\% y el 7,6\% (Tab. 2). Los principales taxones se detallan en la Fig. 2. Este registro polínico permite diferenciar dos grupos de muestras, coincidentes con las facies delimitadas: 
Tabla 2.-Análisis polínico: distribución y abundancia de los taxones determinados (en \%).

\begin{tabular}{|c|c|c|c|c|}
\hline ESPECIES/MUESTRAS & $\mathrm{DN}-1$ & DN-2 & DN-3 & DN-4 \\
\hline Vegetación acuática & 7.61 & 17.58 & 40.8 & 36.46 \\
\hline Typha sp. & 0.99 & 10.94 & 30 & 27.08 \\
\hline Cyperaceae & 6.62 & 5.86 & 6.4 & 6.25 \\
\hline Sparganium sp. & & 0.78 & 3.2 & 3.13 \\
\hline Ranunculus sp. & & & 1.2 & \\
\hline Vegetación higrófila & 5.63 & 1.95 & 5.2 & 11.46 \\
\hline Salix sp. & 1.32 & 0.78 & 1.2 & 4.95 \\
\hline Fraxinus-Phillyrea & 1.32 & & 1.2 & 1.56 \\
\hline Erica sp. & 2.65 & 1.17 & 2 & 4.43 \\
\hline Myrtus communis L. & & & & 0.26 \\
\hline Hypericum sp. & & & 0.8 & 0.26 \\
\hline Potentilla sp. & 0.33 & & & \\
\hline Marismas y Estepas & 46.03 & 53.52 & 9.2 & 5.99 \\
\hline Familia Chenopodiaceae & 43.05 & 36.33 & 8.8 & 5.47 \\
\hline Artemisia sp. & 2.98 & 16.41 & 0.4 & 0.52 \\
\hline Ephedra sp. & & 0.78 & & \\
\hline Vegetación leñosa climatófila & 26.49 & 7.03 & 20.8 & 13.02 \\
\hline Quercus sp. & 4.64 & 1.56 & 5.2 & 5.99 \\
\hline Pinus sp. & 9.27 & 1.95 & 4.4 & 3.65 \\
\hline Olea europaea L. & 7.95 & 1.95 & 5.6 & 2.34 \\
\hline Juniperus sp. & 1.66 & 1.56 & 4.8 & 0.78 \\
\hline Pistacia sp. & 2.65 & & 0.4 & 0.26 \\
\hline Chamaerops humilis L. & 0.33 & & 0.4 & \\
\hline Otras & 8.27 & 15.23 & 16.4 & 25.52 \\
\hline Familia Poaceae & 4.3 & 8.2 & 12 & 13.54 \\
\hline Rumex sp. & 0.99 & 0.78 & 0.8 & 1.3 \\
\hline Familia Lamiaceae & 0.33 & & 0.8 & 2.86 \\
\hline Plantago sp. & 0.99 & & 0.4 & \\
\hline Urtica membranacea Poir. & 0.66 & 0.78 & 0.8 & 0.52 \\
\hline Familia Apiaceae & 0.66 & 0.78 & & 0.52 \\
\hline Familia Inuleae & & 3.13 & & 6.25 \\
\hline Familia Liguliflorae & 0.33 & 0.39 & & 0.26 \\
\hline Familia Anthemideae & & 0.39 & & \\
\hline Familia Fabaceae & & & 1.2 & \\
\hline Familia Liliaceae & & & 0.4 & \\
\hline Echium sp. & & & & 0.26 \\
\hline Cistus sp. & & 0.39 & & \\
\hline Corylus sp. & & 0.39 & & \\
\hline Indeterminadas & 5.96 & 4.69 & 7.6 & 7.55 \\
\hline Número medio de granos $/ \mathrm{mm}^{3}$ & 50 & 80 & 700 & 1400 \\
\hline FACIES & r & 1 & 2 & 2 \\
\hline
\end{tabular}

Facies 1 (muestras DN-1 y DN-2). En el tramo basal de esta facies, el contenido polínico es escaso ( 50 granos $/ \mathrm{mm}^{3}$ ). Predomina la familia Chenopodiaceae (43\%), con Pinus sp. (>9\%),
Olea europea L. ( 8\%) y granos de polen de la familia Cyperaceae $(6,6 \%)$ como principales elementos secundarios de la asociación polínica. A techo de esta facies, disminuye levemente el polen de quenopodiáceas $(36,3 \%)$, en tanto que se incrementa el procedente de Artemisia (16,4\%), de la vegetación acuática (17.6\%; Typha: 11\%) y de la familia Poaceae $(8,2 \%)$.

Facies 2 (muestras DIN-3 y DIN-4). El espectro polínico es bastante similar en las dos muestras analizadas. Está dominado por granos de polen procedentes de la vegetación acuática $(36,4-$ $40,8 \%$ ), entre la que destaca por su abundancia Typha sp. $(27-30 \%)$ y la familia Cyperaceae (6,2-6,4\%). También se observa un aumento de las gramíneas (familia Poaceae: 12-13,5\%) en relación a FA-1, así como porcentajes bajos pero relativamente constantes de quenopodiáceas (5,5-8,8\%), Quercus sp. (5,2-6\%), Pinus sp. (3,6$4,4 \%)$ y Olea europea L. $(2,3-5,6 \%)$.

\section{Diatomeas}

\section{Autoecología}

Los principales parámetros autoecológicos de las especies más abundantes se detallan en la Tab. 3. La mayoría de los taxones tienen un hábitat bentónico o planctónico-bentónico. Limnicola hungarica (Grunow) Round \& Basson es el único taxón específicamente epífito, si bien Diploneis bombus Ehrenb. puede compartir este hábitat.

La mayoría de las especies determinadas pueden vivir tanto en aguas estancadas como fluyentes de climas templados, con un $\mathrm{pH}$ alcalino y una salinidad variable. Solo se ha determinado una especie típica de medios marinos o estuarinos (D. bombus Ehrenb.) y un taxón acidófilo (Eunotia bilunaris (Ehrenb.) Schars.) (Fig. 3).

\section{Distribución}

El registro de diatomeas presenta notables diferencias entre las muestras analizadas, incluso dentro de la misma facies sedimentaria (Tab. 4).

Facies 1. Su tramo basal se caracteriza por la escasez de este grupo (500 individuos $\left./ \mathrm{mm}^{3}\right)$. Está dominado por Ulnaria capitata (Ehrenb.) 


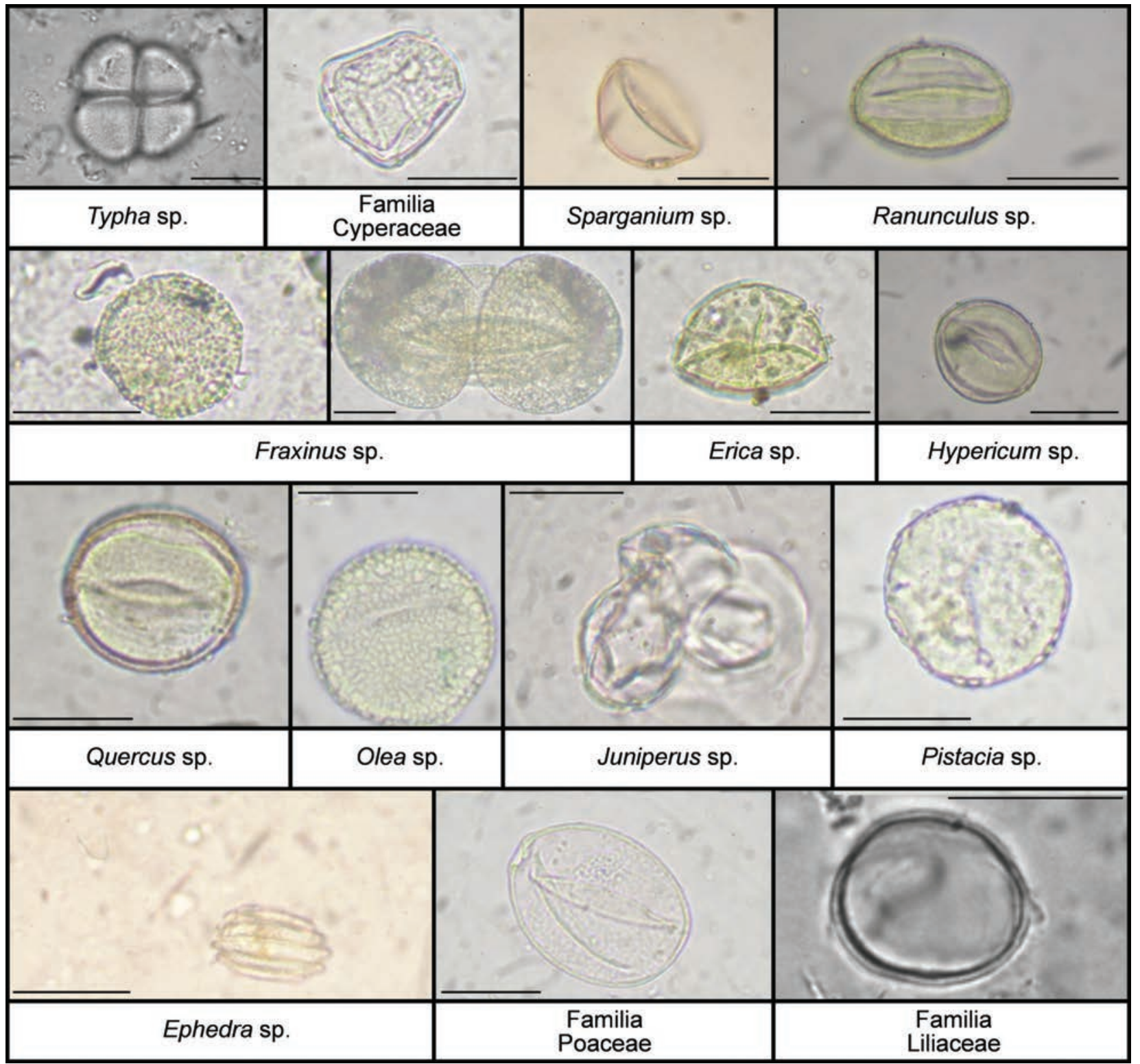

Figura 2.-Análisis polínico: principales taxones determinados.

Compère $(44,3 \%)$ y Cocconeis placentula var. euglypta (Ehrenb.) Grunow (28,9\%). A techo, aumenta de forma notable la concentración de diatomeas $\left(110.000\right.$ individuos $\left./ \mathrm{mm}^{3}\right)$, con una disminución acusada de la primera especie $(14,4 \%)$ y la desaparición de la segunda. Ambas son reemplazadas por Pseudostaurosira brevistriata (Grunow) D.M.Williams \& Round (39,5\%), Staurosira construens Ehrenb. (14,7\%) y Cyclotella meneghiniana Kütz $(7,2 \%)$, en tanto que aumenta levemente sus porcentajes el género Rophalodia (de 6,2\% a $10,4 \%)$.

Facies 2. Su base presenta una disminución de este grupo $\left(35.000\right.$ individuos $\left./ \mathrm{mm}^{3}\right)$. Destaca el repunte de $U$. capitata $(39 \%)$, la disminución de P. brevistriata $(17,8 \%)$ y los porcentajes significativos de $S$. construens $(11,9 \%)$ y Rhopalodia gibberula (Ehrenb.) O. Müll. (8,5\%). Finalmente, no se han encontrado diatomeas en la parte superior de esta facies (DN-4). 
Tabla 3.-Autoecología de las especies determinadas de diatomeas. Los datos han sido extraídos de Buczkó (2007), Piskova et al. (2009), Guiry \& Guiry (2010), Novelo (2012), Perissinotto et al. (2013), López-Fuerte et al. (2015), Tavera et al. (2018) y Niyatbekov \& Barinova (2018).

\begin{tabular}{|c|c|c|c|c|c|}
\hline ESPECIES & HÁBITAT & TEMPERATURA & $\begin{array}{l}\text { OXIGENACIÓN E } \\
\text { HIDRODINÁMICA }\end{array}$ & $\mathrm{pH}$ & SALINIDAD \\
\hline $\begin{array}{l}\text { Cocconeis placentula var. } \\
\text { euglypta (Ehrenb.) Grunow }\end{array}$ & Planctónico-Bentónico & Templada & Aguas estancadas & Alcalófila & Indiferente \\
\hline Cyclotella meneghiniana Kütz. & Planctónico-Bentónico & Templada & Aguas estancadas & Alcalófila & Halófila \\
\hline Cymbella affinis Kütz. & Bentónica & Templada & $\begin{array}{l}\text { Aguas estancadas- } \\
\text { fluyentes }\end{array}$ & Alcalófila & Indiferente \\
\hline Diploneis bombus Ehrenb. & Bentónica-Epífita & Templada-Fría & $\begin{array}{l}\text { Aguas fluyentes: } \\
\text { medios marinos y } \\
\text { estuarinos }\end{array}$ & - & Mesohalófila \\
\hline Epithemia turgida (Ehrenb.) Kütz. & Bentónica & Templada & Aguas estancada & Alcalófila & Indiferente \\
\hline $\begin{array}{l}\text { Eunotia bilunaris (Ehrenb.) } \\
\text { Schars. }\end{array}$ & Bentónica & Templada & $\begin{array}{l}\text { Aguas estancadas- } \\
\text { fluyentes }\end{array}$ & Acidófila & Indiferente \\
\hline $\begin{array}{l}\text { Lemnicola hungarica (Grunow) } \\
\text { Round \& Basson }\end{array}$ & Epífita & Templada & $\begin{array}{l}\text { Aguas estancadas- } \\
\text { fluyentes }\end{array}$ & Alcalófila & Indiferente \\
\hline Melosira varians C. Agardh & Planctónico-Bentónico & Templada & $\begin{array}{l}\text { Aguas estancadas- } \\
\text { fluyentes }\end{array}$ & Alcalófila & Indiferente \\
\hline Navicula radiosa Kütz. & Bentónica & Templada & $\begin{array}{l}\text { Aguas estancadas- } \\
\text { fluyentes }\end{array}$ & Indiferente & Indiferente \\
\hline $\begin{array}{l}\text { Pseudostaurosira brevistriata } \\
\text { (Grunow) D.M.Williams \& } \\
\text { Round }\end{array}$ & Planctónico-Bentónico & Templada & $\begin{array}{l}\text { Aguas estancadas- } \\
\text { fluyentes }\end{array}$ & Alcalófila & Indiferente \\
\hline $\begin{array}{l}\text { Rhopalodia gibba (Ehrenb.) } \\
\text { O.Müll. }\end{array}$ & Bentónica & Templada & $\begin{array}{l}\text { Aguas estancadas- } \\
\text { fluyentes }\end{array}$ & Alcalófila & Indiferente \\
\hline $\begin{array}{l}\text { Rhopalodia gibberula (Ehrenb.) } \\
\text { O.Müll. }\end{array}$ & Bentónica & Templada & Aguas fluyentes & Alcalófila & Mesohalófila \\
\hline Staurosira construens Ehrenb. & Planctónico-Bentónico & Templada & $\begin{array}{l}\text { Aguas estancadas- } \\
\text { fluyentes }\end{array}$ & Alcalófila & Indiferente \\
\hline $\begin{array}{l}\text { Ulnaria capitata (Ehrenb.) } \\
\text { Compère }\end{array}$ & Planctónico-Bentónico & Templada & $\begin{array}{l}\text { Aguas estancadas- } \\
\text { fluyentes }\end{array}$ & Alcalófila & Indiferente \\
\hline
\end{tabular}

\section{Edades}

El rango de edad calibrada del nivel turboso superior (DN-4), muy próximo al contacto erosivo con FA-3, se sitúa entre 390-200 años a.C. (2340-2150 cal. yr BP), en tanto que la edad del ejemplar de Barnea candida es actual-subactual (edad convencional no calibrada: $-667 \pm 34$ años BP).

\section{Discusión: evolución paleoambiental}

El análisis pluridisciplinar de las distintas facies observadas en el perfil estudiado permite efectuar una aproximación a la evolución paleogeográfica de este sector durante el Holoceno superior. Pueden diferenciarse cinco fases (Fig. 4):
Fase 1 (Fig. 4, A-B-C). Este sector estaría ocupado por un sustrato limoso-arcilloso (Facies 1: DN-1) sobre el que se desarrollaría ampliamente la familia Chenopodiaceae, típica de marismas con aportes de aguas saladas. Este carácter salobre también está sustentado por la abundancia de la diatomea Cocconeis placentula var. euglypta (Fig. 3), típica de estos medios (Sabater, 2000). La presencia de pirita implicaría la existencia de condiciones reductoras en el fondo de estas marismas, como consecuencia de la diagénesis de sedimentos ricos en materia orgánica procedente de la degradación de esta vegetación circundante (González et al., 2008). Además, su presencia avalaría el carácter alcalino de las aguas deducido a partir de las asociaciones de diatomeas (Tab. 3), dado que las bacterias responsables de la reducción del sulfato, una reacción implicada en su origen, no operan 


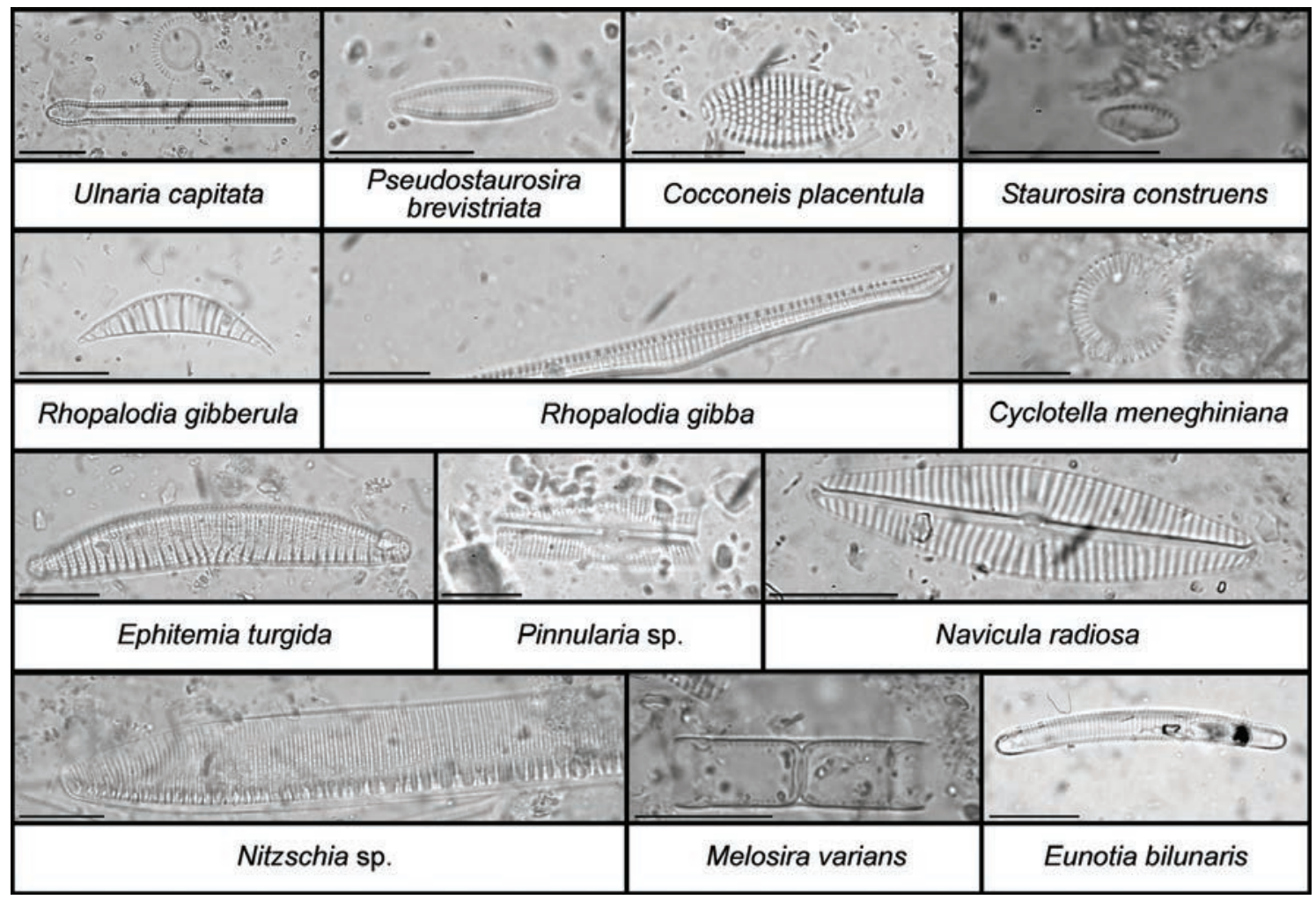

Figura 3.-Diatomeas: principales taxones determinados.

Tabla 4. Diatomeas: distribución y abundancia de los taxones determinados (en \%).

\begin{tabular}{|c|c|c|c|c|}
\hline ESPECIES/MUESTRAS & $\mathrm{DN}-1$ & $\mathrm{DN}-2$ & $\mathrm{DN}-3$ & $\mathrm{DN}-4$ \\
\hline Cocconeis placentula var. euglypta (Ehrenb.) Grunow & 28.87 & & & \\
\hline Cyclotella meneghiniana Kütz. & & 7.19 & 3.72 & \\
\hline Cymbella affinis Kütz. & & 0.65 & & \\
\hline Diploneis bombus Ehrenb. & 3.09 & & 0.37 & \\
\hline Diploneis sp. & & 0.65 & 1.12 & \\
\hline Epithemia turgida (Ehrenb.) Kütz. & 3.09 & 2.61 & 3.72 & \\
\hline Eunotia bilunaris (Ehrenb.) Schars. & 1.03 & 0.33 & 0.37 & \\
\hline Lemnicola hungarica (Grunow) Round \& Basson & & 0.65 & & \\
\hline Melosira varians C.Agardh & & 1.96 & 0.74 & \\
\hline Navicula radiosa Kütz. & & 3.92 & & Fragmentos \\
\hline Nitzschia sp. & 1.03 & 0.65 & 1.12 & \\
\hline Pinnularia sp. & 3.09 & & 1.86 & \\
\hline Pseudostaurosira brevistriata (Grunow) D.M.Williams \& Round & 1.03 & 39.54 & 17.84 & \\
\hline Rhopalodia gibba (Ehrenb.) O.Müll. & 3.09 & 5.88 & 2.97 & \\
\hline Rhopalodia gibberula (Ehrenb.) O.Müll. & 3.09 & 4.58 & 8.55 & \\
\hline Staurosira construens Ehrenb. & 1.03 & 14.71 & 11.9 & \\
\hline Ulnaria capitata (Ehrenb.) Compère & 44.33 & 14.38 & 39.03 & \\
\hline Indeterminadas & 7.22 & 2.29 & 6.69 & \\
\hline Número medio de individuos $/ \mathrm{mm}^{3}$ & 500 & $1,10,000$ & 35,000 & \\
\hline FACIES & & & & 2 \\
\hline
\end{tabular}




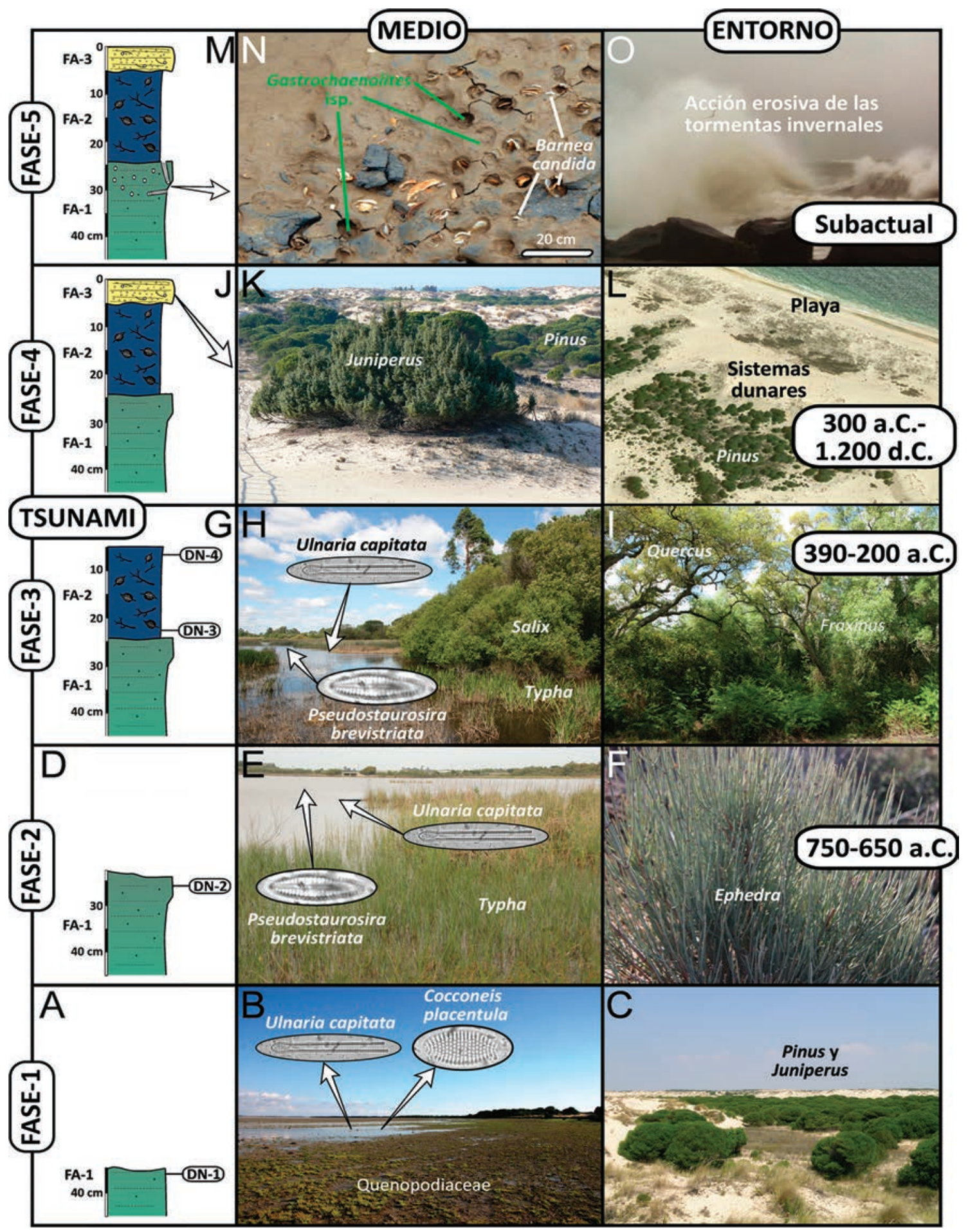

Figura 4.-Evolución paleoambiental del perfil estudiado. 
en condiciones ácidas (Fernández, 1999). Por otro lado, los bajos niveles de polen podrían ser explicados por la baja producción polínica de las comunidades de marisma (Fernández-Illescas et al., 2010) y por la peor conservación del polen en estos medios (Stevenson, 1985a).

La vegetación del entorno estaría constituida por olivos (Olea europea), encinas (Quercus) y pinos (Pinus), que ocupa actualmente una parte significativa del Parque Nacional de Doñana, donde los bosques de la asociación Oleo sylvestris-Quercetum suberis constituirían su clímax. La abundancia de polen de Pinus sp. confirmaría el carácter de acompañante habitual de esta especie en estos bosques, o al menos constataría la existencia de pinares en los trenes de dunas móviles cercanos a la zona (Allier, 1977).

Fase 2 (Fig. 4, D-E-F). Se produce un tránsito a una laguna somera de aguas dulces (Facies 1: DN-2), con desarrollo de helófitos (Typha, Sparganium) en las zonas más profundas. Este aislamiento también queda reflejado en las asociaciones de diatomeas, con la desaparición de especies típicas de medios salobres (C. placentula var. euglypta, Diploneis bombus) y la aparición de taxones propios de aguas estancadas (Fig. 3: Cyclotella meneghiniana) (Niyatbekov \& Barinova, 2018).

Este aislamiento relativo también se detecta en el sedimento del fondo. Se produce una disminución del tamaño medio de grano, con una disminución del porcentaje en arena, probablemente por la interrupción de las conexiones hidrodinámicas con otros medios costeros (Tab. 1). Además, aumenta levemente el contenido en pirita (Tab. 2: del $6 \%$ al $8 \%$ ) y domina la esmectita sobre la illita dentro de los filosilicatos.

En esta fase, tiene su máxima abundancia la asociación Chenopodiaceae-Ephedra-Artemisia (Tab. 2). Esta conjunción indicaría un enfriamiento climático, ya que constituirían una vegetación esteparia (Menéndez Amor \& Florschütz, 1963; Menéndez et al., 2012). Este periodo podría coincidir, de acuerdo con la datación de FA-3, con un evento frío que tuvo lugar hacia los siglos VIIVIII a.C. (2.7 ky cal. BP; Bond et al., 1997).

Fase 3 (Fig. 4, G-H-I). El espectro polínico y los elementos macroscópicos de FA-2 (DN-3 y DN-4) apuntan a la existencia de lagunas permanentes de agua dulce, donde prosperarían las comunidades de helófitos, con abundancia de eneas (Typha) y probablemente de carrizo (Phragmites australis). Los bosques próximos estarían constituidos por fresnedas (Fraxinus, Salix), entre otros. Esta reconstrucción es similar a la efectuada por Stevenson (1985b) en la cercana Laguna de las Madres, con formación de turberas por ausencia de drenaje debido al cierre de la conexión marina por el avance de frentes dunares, si bien el cierre de esta laguna es anterior a la aquí descrita (4200 años BP; Zazo et al., 1996) . Este avance se constata en la granulometría, con un incremento sustancial (Tab. 1: hasta el 13,2\%) del contenido en arenas.

El proceso de turbificación también se aprecia en la mineralogía, con desaparición de la pirita y la presencia de illita e interestratificados irregulares a techo de FA-2 (Tab. 1), que tendrían un origen diagenético (Aróstegui et al., 1993). Es también patente en las diatomeas, donde solo se aprecian fragmentos indiferenciados cerca de la transición a FA-3 (Tab. 3). La progresiva desecación de la laguna y el incremento de los procesos eólicos serían factores desfavorables para el desarrollo de este grupo (Witkowski et al., 2009).

La ausencia de ostrácodos y foraminíferos podría deberse a la presencia de condiciones ácidas temporales en la superficie del sedimento, como consecuencia de los altos niveles de materia orgánica, lo que impediría la preservación de caparazones y valvas de estos grupos (Laprida et al., 2014).

Fase 4 (Fig. 4, J-K-L). La evolución de la turbera finaliza hacia el siglo III a.C., con la implantación posterior de sistemas de dunas (FA-3) sobre los espacios lagunares previos, de acuerdo con la datación obtenida a techo de FA-2 (390-200 a.C.). La edad de este brusco cambio coincide con el final del siglo III a.C., un periodo caracterizado por un tsunami histórico (218 a.C.) y la inundación de Cádiz (210 a.C.), ambos eventos incluidos dentro del catálogo de tsunamis en las costas españolas (Campos, 1991). La progradación posterior de los sistemas dunares circundantes coincidiría con el inicio de la fase regional $\mathrm{H}_{3}$ de Zazo et al. (1994), que se extendería aproximadamente desde el siglo III a.C. hasta el siglo XII d.C. 
Fase 5 (Fig. 4, M-N-O). La edad de los ejemplares de $B$. candida y su aparición como consecuencia de tormentas invernales sugieren que la colonización de FA-1 por este bivalvo vino precedida de un evento erosivo que expuso las arcillas inferiores, ya ligeramente litificadas, y el perfil estudiado en los últimos siglos. Esta zona es erosionada de forma periódica por grandes tormentas invernales, con un ciclo largo de 6-7 años que precede a un ciclo corto posterior de 2-3, de acuerdo con los análisis estadísticos efectuados entre 1960 y 1996 (Ruiz et al., 2005b). Este sector de la playa habría permanecido invadido por el mar durante, al menos, 4 años, de acuerdo con la longitud y densidad de los ejemplares de B. candida (Pinn et al., 2005).

\section{Conclusiones}

El análisis multidisciplinar (textura, mineralogía, paleontología, dataciones) de un perfil situado en el sector occidental del Parque Nacional de Doñana permite perfilar su evolución durante el Holoceno superior. Este estudio delimita las características geológicas de los diferentes paleoambientes deducidos: a) marisma salobre, con abundancia de quenopodiáceas sobre un sustrato arcilloso y un entorno dominado por olivos, encinas y pinos; b) laguna somera de aguas dulces, relativamente aislada, con helófitos en las zonas profundas y quenopodiáceas en las más someras, sometidas a un clima frío; y c) sistemas dunares, caracterizados por su alto contenido de arenas cuarcíferas. Su análisis cronoestratigráfico permite distinguir cinco fases, que abarcan desde una marisma salobre inicial (>2700 años BP) hasta una colonización reciente por bivalvos litófagos.

\section{AGRADECIMIENTOS}

Este trabajo ha sido financiado por los Grupos de Investigación RNM-238 (Paleontología y Ecología Aplicadas), RNM-293 (Geomorfología Ambiental y Recursos Hídricos), RNM-349 (Mineralogía y Geoquímica Ambiental y de la Salud) y RNM-358 (Marismas y Playas) de la Junta de Andalucía, así como por el Plan Propio de Investigación de la Universidad de Huelva. Es una contribución al Centro de Investigación en Patrimonio Histórico, Cultural y Natural (CIPHCN) de la Universidad de Huelva.

\section{Referencias}

Aguirre-Ayerbe, I.; Martínez, J.; Aniel-Quiroga, I.; González-Riancho, P.; Merino, M.; Al-Yahyai, S.; González, M. \& Medina, R. (2018). From tsunami risk assessment to disaster risk reduction-the case of Oman. Natural Hazards and Earth System Sciences, 18: 2241-2260. https://doi.org/10.5194/ nhess-18-2241-2018

Allier, C. (1977). La végétation psammophile du litoral de la Réserve Biologique de Doñana. Monografías ICONA, $18:$ 131-157.

Alonso, C.; Gracia, F.J.; Del Río, L.; Anfuso, G.; Benavente, J.; Martínez, J.A. (2004). Registro morfosedimentario de eventos históricos de alta energía en el litoral Atlántico del Estrecho de Gibraltar (Trafalgar-Tarifa). En: Contribuciones recientes sobre Geomorfología (Benito, G. \& Díez, A., Eds.). SEG-CSIC, Madrid, 263-272.

Alonso, C.; Alonso, C; Gracia, F.J., Rodríguez-Polo, S. \& Martín Puertas, C. (2015). El registro de eventos energéticos marinos en la bahía de Cádiz durante épocas históricas. Cuaternario y Geomorfología, 29: 95-117. https://doi.org/10.17735/ cyg.v29i1-2.29935

Anderson, N.J. (2000). Diatoms, temperature and climatic change. European Journal of Phycology, 35: 307-314. https://doi.org/10.1017/ S0967026200002857

Aniel-Quiroga, I.; González, M.; Álvarez-Gómez, J.A. \& García, P. (2017). Tsunami hazard maps of Spanish coast at national scale from seismic sources. Geophysical Research Abstracts, 19: 15970.

Anfuso, G.; Rangel-Buitrago, N.; Cortés-Useche, C.; Iglesias Castillo, B. \& Gracia, F.J. (2016). Characterization of storm events along te Gulf of Cádiz (eastern central Atlantic Ocean). International Journal of Climatology, 36: 3690-3707. https:// doi.org/10.1002/joc.4585

Araujo, N.; Moraes, C.A.; Cohen, M.C.L; Alves, I.C.; Franca, M.C.; Pessenda, L.C.R.; Francisquini, M.I.; Bendassolli, J.A.; Macario, K. \& Mayle, F. (2017). The Impacts of the Middle Holocene High Sea-Level Stand and Climatic Changes on Mangroves of the Jucuruçu River, Southern Bahia Northeastern Brazil. Radiocarbon, 59: 215-230. https://doi.org/10.1017/RDC.2017.6

Arias, J.P.; Bronfman, N.C.; Cisternas, P.C. \& Repetto, P.B. (2017). Hazard proximity and risk perception of tsunamis in coastal cities: Are people able to identify their risk?. Plos One, 12: e0186455. https:// doi.org/10.1371/journal.pone.0186455

Aróstegui, J.; Nieto, F.; Ortega-Huertas, M.; Velasco, F. \& Zuluaga, M.C. (1993). Mineralogía de arcillas y grado de diagénesis del Cretácico Inferior, en el flanco sur del anticlinorio de Bilbao. Estudios 
Geológicos, 49: 277-286. https://doi.org/10.3989/ egeol.93495-6355

Barahona, E. (1974). Arcillas de ladrillería de la provincia de Granada: evaluación de algunos ensayos de materias primas. Tesis Doctoral, Universidad de Granada, 398 pp.

Blázquez, A.M.; Rodríguez-Pérez, A.; Torres, T. \& Ortiz, J.E. (2017). Evidence for Holocene sea level and climate change from Almenara marsh (western Mediterranean). Quaternary Research, 88: 206-222. https://doi.org/10.1017/qua.2017.47

Bond, G.; Showers, W.; Cheseby, M.; Lotti, R.; Almasi, P.; de Menocal, P.; Priore, P.; Cullen, H.; Hajdas, I. \& Bonani, G. (1997). A pervasive millennial-scale cycle in North Atlantic Holocene and Glacial climates. Science, 278: 1257-1266. https://doi. org/10.1126/science.278.5341.1257

Borja, F. (2013). La desembocadura del Guadalquivir durante la segunda mitad del Holoceno. Síntesis paleogeográfica. En : El Asentamiento histórico de Valencina de la Concepción (Sevilla) : Investigacion y tutela en el 150 aniversario del descubrimiento de La Pastora (García, L., Vargas, J.M., Hurtado, V., Ruiz, T. \& Cruz-Auñón, R., Eds). Universidad de Sevilla, Sevilla, 93-112.

Borrego, J., Morales, J.A. \& Pendón, J.G. (1993). Elementos morfodinámicos responsables de la evolución reciente del estuario bajo del río Guadiana (Huelva). Geogaceta, 11: 86-89.

Borrego, J., Ruiz, F.; González-Regalado, M.L.; Pendón, J.G. \& Morales, J. A. (1999). The Holocene transgression into the estuarine central basin of the Odiel River mouth (Cádiz Gulf, SW Spain): lithology and faunal assemblages. Quaternary Science Reviews, 18: 769-788. https://doi. org/10.1016/S0277-3791(97)00085-1

Buczkó, K. (2007). The occurrence of the epiphytic diatom Lemnicola hungarica on different European Lemnaceae species. Fottea, Olomouc, 7: 77-84. https://doi.org/10.5507/fot.2007.007

Campos, M.L. (1991). Tsunami hazard on the Spanish coasts of the Iberian Peninsula. The Science of the Tsunami Hazard, 9: 83-90.

Castele, B.; Dodet, G.; Masselink, G. \& Scott, T. (2018). Increased winter-mean wave height, variability, and periodicity in the Northeast Atlantic over 19492017. Geophysical Research Letters, 45: 35863596. https://doi.org/10.1002/2017GL076884

Civis, J.; Sierro, F.J.; González Delgado, J.A.; Flores, J.A.; Andrés, I.; Porta, J. \& Valle, M.F. (1987). El Neógeno marino de la provincia de Huelva: antecedentes y definición de las unidades litoestratigráficas. In: Paleontología del Neógeno de Huelva (Civis, J., Ed.), Ediciones de la Universidad de Salamanca, Salamanca, 9-21.

Chase, B.M. \& Quick, L.J. (2018). Influence of Agulhas forcing of Holocene climate change in
South Africa's southern Cape. Quaternary Research, 90: 303-309. https://doi.org/10.1017/qua.2018.57

Cohen, M.C.L.; Lara, R.J.; Cuevas, E.; Oliveras, E.M. \& Sternberg, L.S. (2016). Effects of sea-level rise and climatic changes on mangroves from southwestern littoral of Puerto Rico during the middle and late Holocene. Catena, 143: 187-200. https://doi. org/10.1016/j.catena.2016.03.041

Dabrio, C.J.; Zazo, C.; Lario, J.; Goy, J.L.; Sierro, F.J.; Borja, F.; González, J.A. \& Flores, J.A. (1999). Sequence stratigraphy of Holocene incised valley fills and coastal evolution in the Gulf of Cádiz (southern Spain). Geologie in Mijnbouwn, 77: 263-281. https://doi.org/ 10.1023/A:1003643006015

Dabrio, C.J.; Zazo, C.; Goy, J.L.; Sierro, F.J.; Borja, F.; Lario, J.; González-Delgado,J.; Flores, J.A. (2000). Depositional history of estuarine infill during the last postglacial transgression (Gulf of Cadiz, Southern Spain). Marine Geology, 162: 381-404. https://doi.org/10.1016/S0025-3227(99)00069-9

Fanget, A.S.; Bassetti, M.A.; Fontanier, C.; Tudryn, A. \& Berné, S. (2016). Sedimentary archives of climate and sea-level changes during the Holocene in the Rhône prodelta (NW Mediterranean Sea). Climate Paste, 12: 2161-2179. https://doi. org/10.5194/cp-12-2161-2016

Fernández, M.L. (1999). Contribución al estudio de las marismas de los ríos Xubia y Ferrerías. Tesis Doctoral, Universidad de A Coruña, $350 \mathrm{pp}$.

Fernández-Illescas, F.; Nieva, F.J.J.; Silva, I.; Tormo, R. \& Muñoz, A.M. (2010). Pollen production of Chenopodiaceae species at habitat and landscape scale in Mediterranean salt marshes: An ecological and phenological study. Review of Palaeobotany and Palynology, 161: 127-136. https://doi.org/10.1016/j.revpalbo.2010.03.006

Font, E., Veiga-Pires, C., Pozo, M., Nave, S., Costa, S., Ruiz, F., Abad, M., Simoes, N., Duarte, S. \& Rodríguez Vidal, J. (2013). Benchmarks and sediment source(s) of the 1755 Lisbon tsunami deposit at Boca do Rio Estuary. Marine Geology, 343: 1-14. https://doi.org/10.1016/j.margeo.2013.06.008

Galbis, R.J. (1932). Catálogo sísmico de la zona comprendida entre los meridianos $58 \mathrm{E}$ y $20 \mathrm{~W}$ de Greenwich y los paralelos $45^{\circ}$ y $25^{\circ} \mathrm{N}$. Dirección General del Instituto Geográfico, Catastral y de Estadística, Madrid.

González, C.; Dupont, L.M., Behling, H. \& Wefer, G. (2008). Neotropical vegetation response to rapid climate changes during the last glacial period: Palynological evidence from the Cariaco Basin. Quaternary Research, 69: 217-230. https://doi. org/10.1016/j.yqres.2007.12.001

Gràcia, E.; Vizcaino, A.; Escutia, C.; Asioli, A.; Rodés, A., Pallá, R.; García-Orellana, J.; Lebreiro, S. \& Goldfinger, C. (2010). Holocene earthquake 
record offshore Portugal (SW Iberia): testing turbidite paleoseismology in a slow-convergence margin. Quaternary Science Reviews, 29: 1156-1172. https://doi.org/10.1016/j.quascirev.2010.01.010

Guiry, M.D. \& Guiry, G.M. (2010). AlgaeBase. Worldwide electronic publication, National University of Ireland. Galway. http://www.algaebase.org

Gutiérrez-Mas, J.M. \& Mas, R. (2013). Record of very high energy events in Plio-Pleistocene marine deposits of the Gulf of Cádiz (SW Spain): facies and processes. Facies, 59: 679-701. https://doi. org/10.1007/s10347-012-0344-y

Gutiérrez-Mas, J.M.; Juan, C. \& Morales, J.A. (2009). Evidence of high-energy events in shelly layers interbedded in coastal Holocene sands in Cádiz Bay (south-west Spain). Earth Surface Processes and Landforms, 34: 810-823. https://doi. org/10.1002/esp. 1770

Kelly, M.G; Bennion, H.; Cox, E.J.; Goldsmith, B.; Jamieson, J.; Juggins, S.; Mann, D.G. \& Telford, R.J. (2005). Common freshwater diatoms of Britain and Ireland: an interactive key. Versión CD-ROM. Environment Agency. Bristol.

Laprida, C.; Massaferro, J.; Ramón, M.J.; Cuminsky, G. (2014). Paleobioindicadores del fin del mundo: ostrácodos y quironómidos del extremo sur de Sudamérica en ambientes lacustres Cuaternarios. Latin American Journal of Sedimentary Basin Analysis, 21: 97-117.

Lario, J.; Luque, L.; Zazo, C.; Goy, J.L.; Spencer, C.; Cabero, A.; Bardají, T.; Borja, F.; Dabrio, C.J.; Civis, J.; González-Delgado, J.A.; Borja, C.; Alonso-Azcárate, J. (2010). Tsunami vs. storm surge deposits: a review of the sedimentological and geomorphological records of extreme wave events (EWE) during the Holocene in the gulf of Cadiz, Spain, Zeitschrift für Geomorphologie, 54: 301-316. https://doi. org/10.1127/0372-8854/2010/0054S3-0029

Lario, J.; Zazo, C.; Goy, J.L., Silva, P.G., Bardají, T.; Cabero, A.; Dabrio, C.J. (2011). Holocene palaeotsunami catalogue of SW Iberia. Quaternary International, 242: 196-200. https://doi.org/10.1016/j. quaint.2011.01.036

Leventer, A.; Crosta, X. \& Pike, J. (2010). Holocene marine diatom records of environmental change. In: The Diatoms: Applications for the Environmental and Earth Sciences (Smol, J.P. \& Stoermer, E.F., Eds.), Cambridge University Press, Cambridge, 400-422. https://doi.org/10.1017/ CBO9780511763175.022

López-Fuerte, F.O.; Siqueiros-Beltrones, D.A. \& Yabuer, R. (2015). First record of benthic diatoms (Bacillariophyceae and Fragilariophyceae) from Isla Guadalupe, Baja California, Mexico. Revista Mexicana de Biodiversidad, 86: 281-292. https:// doi.org/10.1016/j.rmb.2015.04.016
Luque, L., Lario, J., Zazo, C., Goy, J.L., Dabrio, C.J. \& Silva, P.G. (2001). Tsunami deposits as palaeoseismic indicators: examples from the Spanish coast. Acta Geologica Hispanica, 3-4: 197-211.

Luque, L., Lario, J., Civis, J., Silva, P.G., Zazo, C., Goy, J.L. \& Dabrio, C.J. (2002). Sedimentary record of a tsunami during Roman times, Bay of Cadiz, Spain. Journal of Quaternary Science, 17, 623-631. https://doi.org/10.1002/jqs.711

Martínez Solares, J.M. (2005). Los tsunamis en el contexto de la Península Ibérica. Enseñanza de las Ciencias de la Tierra, 13: 52-59.

Martins, J.M.M. \& Soares, A.M.M. (2013). Marine radiocarbon reservoir effect in Southern Atlantic Iberian coast. Radiocarbon, 55: 1123-1134. https://doi.org/10.1017/S0033822200048037

Mayoral, E. \& Pendón, J.G. (1986-87). Icnofacies y sedimentación en zona costera. Plioceno superior (?), litoral de Huelva. Acta Geológica Hispánica, 21-22: 507-513.

Menanteau, L. (1979). Les Marismas du Guadalquivir. Example de transformation d'un pausage alluvial au cours du Quaternaire récent. Thèse 3er cycle, Université Paris-Sorbonne, 154 pp.

Menéndez Amor, J. \& Florschütz, F. (1963). Sur les éléments steppiques dans la végétation quaternaire de l'Espagne. Boletín de la Real Sociedad Española de Historia Natural, 61: 121-133.

Menéndez, M.; Ayarzagüena, M.; Fano, M.A.; García, E.; Jordá, J.F.; Mas, M.; Mingo, A.; Quesada, J.M. \& Rosas, A. (2012). Prehistoria Antigua de la Península Ibérica. Universidad Nacional de Educación a Distancia, Madrid, 656 pp.

Niyatbekov, T. \& Barinova S.S. (2018). Bioindication of aquatic habitats with diatom algae in the Pamir Mountains, Tajikistan. MOJ Ecology \& Environmental Sciences, 3: 117-120.

Novelo, E. (2012). Bacillariophyta. Flora del Valle de Tehuacán-Cuicatlán, 102: 1-230.

Perissinotto, R.; Bate, G.C. \& Muir, D.G. (2013). Microalgae. In: Ecology and Conservation of Estuarine Ecosystems (Perissinotto, R., Stretch, D.D. \& Taylor, R.H., Eds.), Cambridge University Press, Cambridge, 187-208. https://doi.org/10.1017/ CBO9781139095723.012

Pinn, E.H.; Richardson, C.A.; Thompson, R.C. \& Hawkins, S.J. (2005). Burrow morphology, biometry, age and growth of piddocks (Mollusca: Bivalvia: Pholadidae) on the Routh coast of England. Marine Biology, 147: 943-953. https:// doi.org/10.1007/s00227-005-1582-0

Piskova, A.; Grygar, T.M.; Kulichova, J. \& Oberhänsli, H. (2009). Diatom assemblage variations in the Aral Sea Core C2/2004 over the past two millennia. Journal of the Czech Phycological Society, 9: 333-342. https://doi.org/10.5507/fot. 2009.032 
Pouzet, P.; Maanan, M.; Piotrowska, N.; Batlzer, A.; Stéphan, P. \& Robin, M. (2018). Chronology of Holocene storm events along the European Atlantic coast. Progress in Physical Geography: Earth and Environment, 42: 431-450. https://doi. org/10.1177/0309133318776500

Prieto, A.R.; Mourelle, D.; Peltier, W.R.; Drummond, R.; Vilanova, I. \& Ricci, L. (2017). Relative sea-level changes during the Holocene in the Río de la Plata, Argentina and Uruguay: A review. Quaternary International, 442: 35-49. https://doi. org/10.1016/j.quaint.2016.02.044

Putra, P.S. (2018). Tsunami sediments and their grain size characteristics. IOP Conference Series Earth and Environmental Science, 118: 012035. https:// doi.org/10.1088/1755-1315/118/1/012035

Quick, L.J.; Chase, B.M.; Wündsch, M.; Kirsten, K.L.; Chevalier, M.; Mäusbacher, R.; Meadows, M.E. \& Haberzetti, T. (2018). A high-resolution record of Holocene climate and vegetation dynamics from the southern Cape coast of South Africa: pollen and microcharcoal evidence from Eilandvlei. Journal of Quaternary Science, 33: 487-500. https://doi.org/10.1002/jqs.3028

Recio, J.M.; Díaz del Olmo, F.; Borja, C.; Borja, F.; Cámara, R. (2009). Changes in the Recent Pedogenesis. Role of the Hydromorphism in Two Sma1l-Lakes of the Doñana National Park (Huelva, SW Spain). Ecology and Noospherology, 20: 145-147.

Reicherter, K. (2001). Paleoseismological advances in the Granada Basin (Betic Cordilleras, southern Spain). Acta Geologica Hispanica, 36: 267-281.

Rodríguez Vidal, J.; Ruiz, F.; Cáceres, L. M.; Abad, M.; González-Regalado, M.L.; Pozo, M.; Carretero, M.I.; Monge, A.M. \& Gómez, F. (2011a). Geomarkers of the 218-209 BC Atlantic tsunami in the Roman Lacus Ligustinus (SW Spain): A palaeogeographical approach. Quaternary International, 242: 201-212. https://doi.org/10.1016/j. quaint.2011.01.032

Rodríguez Vidal, J.; Cáceres, L. M.; Abad, M.; Ruiz, F.; González-Regalado, M.L.; Finlayson, C.; Fynlayson, G.; Fa, D.; Rodríguez-Llanes, J. M. \& Bailey, G. (2011b). The recorded evidence of AD 1755 Atlantic tsunami on the Gibraltar coast. Journal of Iberian Geology, 37: 177-193. https:// doi.org/10.5209/rev_JIGE.2011.v37.n2.7

Rubin, C.M.; Horton, B.P.; Sieh, K.; Pilarczyk, J.E.; Daly, P.; Ismail, N. \& Parnell, A. (2017). Highly variable recurrence of tsunamis in the 7,400 years before the 2004 Indian Ocean tsunami. Nature Communications, 8: 16019. https://doi. org/10.1038/ncomms16019

Ruiz, F.; Rodríguez Ramírez, A.; Cáceres, L.M.; Rodríguez Vidal, J.; Carretero, M.I.; Clemente, L.; Muñoz, J.M.; Yañez, C. \& Abad, M. (2004). Late
Holocene evolution of the southwestern Doñana Nacional Park (Guadalquivir Estuary, SW Spain): a multivariate approach. Palaeogeography, Palaeoclimatology, Palaeoecology, 204: 47-64. https://doi.org/10.1016/S0031-0182(03)00721-1

Ruiz, F.; Rodríguez-Ramírez, A.; Cáceres, L.M.; Rodríguez Vidal, J.; Carretero, M.I.; Abad, M.; Olías, M. \& Pozo, M. (2005a). Evidence of highenergy events in the geological record: MidHolocene evolution of the southwestern Doñana National Park (SW Spain). Palaeogeography, Palaeoclimatology, Palaeoecology, 229: 212-229. https://doi.org/10.1016/j.palaeo.2005.06.023

Ruiz, F.; Abad, M.; Rodríguez-Ramírez, A.; Cáceres, L.M.; Rodríguez Vidal, J.; Pino, R. \& Muñoz, J.M. (2005b). A statistical approach to the critical storm period analysis. In: The Water Enciclopedia (Lehr, J.H., Keeely, J., Eds.), John Wiley \& Sons, New York, 1-5.

Ruiz, F.; Abad, M.; Rodríguez Vidal, J.; Cáceres, L.M.; González-Regalado, M.L.; Carretero, M.I.; Pozo, M \& Gómez Toscano, F. (2008). The geological record of the oldest historical tsunamis in southwestern Spain. Rivista Italiana di Paleontologia e Stratigrafia, 114: 147-156.

Ruiz, F.; Rodríguez Vidal, J.; Abad, M.; Cáceres, L.M.; Carretero, M.I.; Pozo, M.; Rodríguez-Llanes, J.M.; Gómez-Toscano, F.; Izquierdo, T.; Font, E. \& Toscano, A. (2013). Sedimentological and geomorphological imprints of Holocene tsunamis in Southwestern Spain: An approach to establish the recurrence period. Geomorphology, 203: 97-104. https://doi.org/10.1016/j.geomorph.2013.09.008

Sabater, S. (2000). Diatom communities as indicators of environmental stress in the Guadiamar River, SW Spain, following the major mine tailings spill. Journal of Applied Phycology, 12: 113-124. https://doi.org/10.1023/A:1008197411815

Salvany, J.M. \& Custodio, E. (1995). Características litoestratigráficas de los depósitos plio-cuaternarios del bajo Guadalquivir en el área de Doñana: implicaciones hidrogeológicas. Revista de la Sociedad Geológica de España, 8: 21-31.

Salvany, J.M., Mediavilla, C. \& Rebollo, A. (2010). Las formaciones Plio-Cuaternarias de El Abalario, en el litoral de la provincia de Huelva. Estudios Geológicos, 66: 209-225. https://doi.org/10.3989/ egeol.39953.072

Scheffers, F. \& Kelletat, D. (2005). Boulder deposits on the southern Spanish Atlantic coast: possible evidence for the 1755 AD Lisbon Tsunami. The Science of the Tsunami Hazards, 23: 25-38.

Stevenson, A.C. (1985a). Studies in the vegetational history of SW Spain. I. Modern Pollen Rain in the Doñana National Park, Huelva. Journal of Biogeography, 12: 243-268. https://doi. org/10.2307/2844998 
Stevenson, A.C. (1985b). Studies in the Vegetational History of SW Spain. II. Palynological investigations at Laguna de las Madres, SW Spain. Journal of Biogeography, 12: 293-314. https://doi. org/10.2307/2844863

Tavera, R.; Novelo, E. \& Orozco, C. (2018). Las diatomeas del Parque Ecológico de Xochimilco, México ¿Qué tan importante es la flora de un grupo de un pequeño lugar?. Cymbella, 4: 3-46.

Toja, J.; López, T. \& Gabellone, N. (1991). Successional changes in two dune ponds (Doñana National Park). Verh. Int. Verein. Limnol., 24: 1556-1559.

Valdés, B., Díez, M.J. \& Fernández, I. (1987). Atlas polínico de Andalucía Occidental. Universidad de Sevilla \& Diputación de Cádiz (Eds), Sevilla, 450 pp.

Vanney, J.R. (1970). L'hydrologie du Bas Guadalquivir. Ed. CSIC-Consejo Superior de Investigaciones Científicas-. Madrid.

Vanney, J.R.; Menanteau, L. (1985). Physiographic map of the Atlantic littoral of Andalousia 1:50.000. Junta de Andalucía.

Witkowski, A.; Cedro, B.; Kierzek, A. \& Baranowski, D. (2009). Diatoms as a proxy in reconstructing the Holocene environmental changes in the south-western Baltic Sea: the lower Rega River Valley sedimentary record. Hydrobiologia, 631: 155-172. https://doi.org/10.1007/s10750-009-9808-7

Zazo, C.; Goy, J.L.; Somoza, L.; Dabrio, C.J.; Belluomini, G.; Improta, S.; Larío, J.; Bardají, T. \& Silva, P.G. (1994). Holocene Sequence of Sea-Level Fluctuations in Relation to Climatic Trends in the Atlantic-Mediterranean Linkage Coast. Journal of Coastal Research, 10: 933-945.

Zazo, C.; Lezine, A.M.; Borja, F.; Denèfle, M.; Dabrio, C. J.; Lario, J.; Rodríguez Vidal, J.; Goy, J.L.; Bardají, T.; Díaz del Olmo, F.; Cáceres, L.; Clemente, L.; Baeteman, C. \& Rodríguez, A. (1996). Holocene coastal progradation changes and peat bog development in SW Spanish coast. Mediterranean and Black Sea Shorelines Newsletter, 18: 13-17.

Zazo, C., Dabrio, C.J., Goy, J.L., Lario, J., Cabero, A., Silva, P.G., Bardají, T., Mercier, N., Borja, F. \& Roquero, E. (2008). The coastal archives of the last $15 \mathrm{ka}$ in the Atlantic-Mediterranean Spanish linkage area: sea level and climatic changes. Quaternary International, 181: 72-87. https://doi. org/10.1016/j.quaint.2007.05.021 